\title{
The minimum quantity of lubricant (MQL) technique in the surface grinding of steel
}

\author{
E. C. Bianchi ${ }^{1}$, R. S. Destro ${ }^{1}$, M. H. Oikawa ${ }^{1}$, R. M. Sousa ${ }^{1}$, \\ R. C. Canarim ${ }^{1}$, R. F. Damasceno ${ }^{1}$, P. R. Aguiar $^{2}$ \\ \& M. C. Sampaio Alves ${ }^{3}$ \\ ${ }^{1}$ Department of Mechanical Engineering, \\ UNESP - São Paulo State University, Brazil \\ ${ }^{2}$ Department of Electrical Engineering, \\ UNESP - São Paulo State University, Brazil. \\ ${ }^{3}$ Department of Wood Engineering, UNESP - São Paulo State, Brazil
}

\begin{abstract}
This research aimed to analyze the viability of the minimum quantity of lubricant (MQL) technique towards different methods of lubri-refrigeration in surface grinding of steel, considering process quality, wheel life and the viability of using cutting fluids. The proposal methods were the conventional (abundant fluid flow), the minimum quantity lubrication (MQL) and the optimized method with Webster nozzle (rounded). This analysis was carried out in equal machining conditions, through the assessment of variables such as grinding force, surface roughness, $G$ ratio (volume of removed material/volume of wheel wear), and microhardness. The results showed the possibility of improvement of the grinding process. Besides, there is the opportunity for production of high quality workpieces with lower costs. The MQL technique showed efficiency in machining with lower depths of cut. The optimized method with Webster nozzle applies the fluid in a rational way, without considerable waste. Hence, the results show that industry can rationalize and optimize the application of cutting fluids, avoiding inappropriate disposal, inadequate use and consequently environment pollution.
\end{abstract}

Keywords: grinding, cutting fluids, MQL, optimization. 


\section{Introduction}

Grinding is a very complex machining process, with many characteristic parameters influencing each other [1]. Its main characteristic is the material removal by the contact between a tool containing abrasive grains (grinding wheel) and the workpiece surface. Each grain of the grinding wheel topographic structure removes a small quantity of material, providing the work surface with a better finishing [2].

According to Hassui and Diniz [3], grinding is the ideal process for a workpiece which needs good surface, dimensional and geometric qualities. Due to these requirements, the process is usually one of the last steps in the machining chain. When the workpiece reaches the grinding stage, it already has high aggregated value from the previous procedures. This makes a possible failure during the finishing process with consequent rejection very expensive and undesirable.

Process industries are seeking for higher production, cost reduction, flexibility, better dimensional and surface quality. This is becoming more and more important to these industries in order to keep, or even increase their market shares in global economy [4]. Grinding is a finishing process, characterized by obtaining results such as precise dimensional tolerance and high superficial quality. However, a limiting factor is the excessive heat generation on the cutting interface. This excessive heat can cause thermal damages to the workpiece, undesirable in a finishing process. Thus, to minimize heat generation, fluids are used to cool and lubricate the cutting zone [5].

In grinding processes, the function of cutting fluids is to remove the excessive heat generated during the cutting operation, lubricate the region where the contact occurs, expel the excess of chips formed, keep the grinding wheel clean, and minimize corrosion on the material involved [6].

Nevertheless, cutting fluids are dangerous substances for the environment. Their disposal is considerably expensive and involves hard procedures. Besides, those substances can cause several diseases after prolonged exposures, such as dermatitis, problems in the respiratory and digestive system, and sometimes even cancer [6].

As shown, cutting fluids play an important role in the productive process, and their use results, most of the times, in a longer life cycle of the tool and in better quality of the machined workpiece. However, their application brings along a few issues, such as difficulties and high cost with recycling and disposal, environmental pollution and chronic diseases for the people exposed. Because of this, a lot of research has been done in order to avoid or minimize cutting fluids use in grinding processes, but without losing the benefits these fluids cause, mainly related to the tool life cycle and the workpiece quality [7].

This paper seeks to analyze lubrication-cooling alternative methods to the conventional method, making it possible a more rational use of cutting fluids, without any losses concerning the quality parameters. These methods are, mainly, the minimum quantity lubrication (MQL), and a fluid application optimized method, with a nozzle proposed by Webster [8]. Through 
experimentation and analysis of obtained data, the purpose is to verify if the MQL technique can be considered as an alternative to fluid application in grinding, keeping the standards for dimensional and surface quality, characteristics of the process studied. Besides, it is proposed to analyze whether the optimized method with Webster [8] nozzle can be an alternative to the conventional method of application in terms of cutting fluid usage.

In this research, the grinding wheel peripheral velocity and the workpiece velocity were kept constant, and the depth of cut varied in $0.02 \mathrm{~mm}, 0.05 \mathrm{~mm}$ and $0.08 \mathrm{~mm}$. This choice was made in order to simulate three different conditions of grinding: a smooth one $(0.02 \mathrm{~mm})$, typical of finishing; a severe one $(0.08 \mathrm{~mm})$; and an intermediate one $(0.05 \mathrm{~mm})$. Hence, the adopted values can summarize the conditions of grinding in industries. These values were obtained by means of experimental testing. Besides that, the grinding machine used allows a better control of the depth of cut than the other parameters.

\section{Materials and methods}

The equipment set consists of a SulMecânica 1055E surface grinder; a vitrified superabrasive grinding wheel CBN $(350 \mathrm{~mm}$ external diameter, $127 \mathrm{~mm}$ internal diameter, $20 \mathrm{~mm}$ width, with $5 \mathrm{~mm}$ thickness of abrasive material), code SNB151Q12VR2; AISI 4340 workpiece, tempered and quenched (54 HRc average hardness), rectangular shape, 100×200×10mm dimensions. The dressing operation was kept constant, using a multigranular dresser, which did not influence the output variables of the process.

The cutting fluid utilized in the conventional and optimized methods was semi-synthetic soluble oil, with concentration of $5 \%$ of fluid in the emulsion, as recommended by the manufacturer. In the conventional method, this fluid is plentifully applied on the contact interface, in a flow rate of approximately $27.51 / \mathrm{min}$. In the optimized method, a pump makes it possible to apply the fluid at a velocity of $32 \mathrm{~m} / \mathrm{s}$, the same as the peripheral of the grinding wheel. In this case, the pressure used was about 8bar, and flow rate is the same as the one used in the conventional method $(27.51 / \mathrm{min})$.

The MQL technique consists of atomizing small quantities of fluid in a compressed air line. The MQL system is composed of: compressor, pressure regulator, air flow meter, dosing device and nozzle. It allows a fine adjustment of lubricant/air volume independently, by means of a needle type register. The cutting fluid used was biodegradable vegetal oil with additives for extreme pressure, severe operations and antioxidants. In this experiment, the air flow was pressured nearly 8 bar, and cutting fluid rate was $100 \mathrm{ml} / \mathrm{h}$.

The optimized method with Webster [8] nozzle requires a special design with rounded surfaces, which can reduce turbulence flow of the cutting fluid. This nozzle was manufactured as close as the author indicates in his paper. This method also requires a pump, in order to apply the fluid at the cutting zone.

With the equipment ready, preliminary experiments were carried out, to define grinding parameters. Keeping the workpiece velocity at $0.0033 \mathrm{~m} / \mathrm{s}$ and 
the grinding wheel peripheral velocity at $32 \mathrm{~m} / \mathrm{s}$, both constant, the depth of cut was varied in $0.02 \mathrm{~mm}, 0.05 \mathrm{~mm}$ and $0.08 \mathrm{~mm}$.

The end criterion was the specific volume of the material removed, adopted $5000 \mathrm{~mm}^{3} / \mathrm{mm}$. Since there were three different depths of cut, the interval to obtain the specific volume was different in each situation.

The tangential cutting force was measured through the determination of electric power consumed by the engine of the grinder spindle, using an electronic module and a board of data gathering, manipulated by a data gathering program, based on the software LabView, from National Instruments ${ }^{\circledR}$.

Surface roughness data were obtained measuring $R_{a}$, parameter with a rugosimeter Taylor Hobson Surtronic ${ }^{3+}$, at each $1000 \mathrm{~mm}^{3} / \mathrm{mm}$ of removed material.

The grinding wheel wear measurement was made using specimen made of AISI 1045 steel for impress the wheel. The impression made was measured by a Tesa Micro-Hite 2D Direct Computer Control (DCC) coordinate measuring machine.

Vickers microhardness was also determined. To obtain this measure, a Buehler 1600-6300 meter was used.

For each depth of cut, three different lubrication-cooling methods were tested, the conventional, the MQL and the optimized method application with Webster nozzle. For each method, the experiments were repeated three times. Thus, a total of 27 experiments were carried out.

\section{Results and discussions}

In this topic, the gathered data was analyzed. It is important to remember that the workpiece and the grinding wheel peripheral velocities were kept constant, only the depth of the cut varied in $0.02 \mathrm{~mm}, 0.05 \mathrm{~mm}$ and $0.08 \mathrm{~mm}$.

\subsection{Tangential cutting force}

Figure 1 shows the tangential cutting force behaviour along the experiment for the three depths of cut. It can be observed that in the MQL technique at 0.02 and $0.05 \mathrm{~mm}$ depth of cut the cutting force is lower than that observed in the conventional and optimized methods. This can be explained by the fact that the MQL, under those conditions, allows a more effective lubrication in the cutting zone, overcoming the air barrier generated by the grinding wheel through its movement. This way, the strength spent on the friction between the workpiece and the tool is minimized. Similar condition is observed in the optimized method, whose behaviour is quite similar to MQL.

At $0.08 \mathrm{~mm}$ depth of cut, the force observed in the MQL is the highest. That grinding condition is the most severe among the ones analyzed, which can be confirmed by an increase in the cutting force. The fact that the forces in the MQL are greater refers to the difficulties in ensuring efficient lubrication. Under these more severe conditions of cutting, high stresses are present. Besides the 


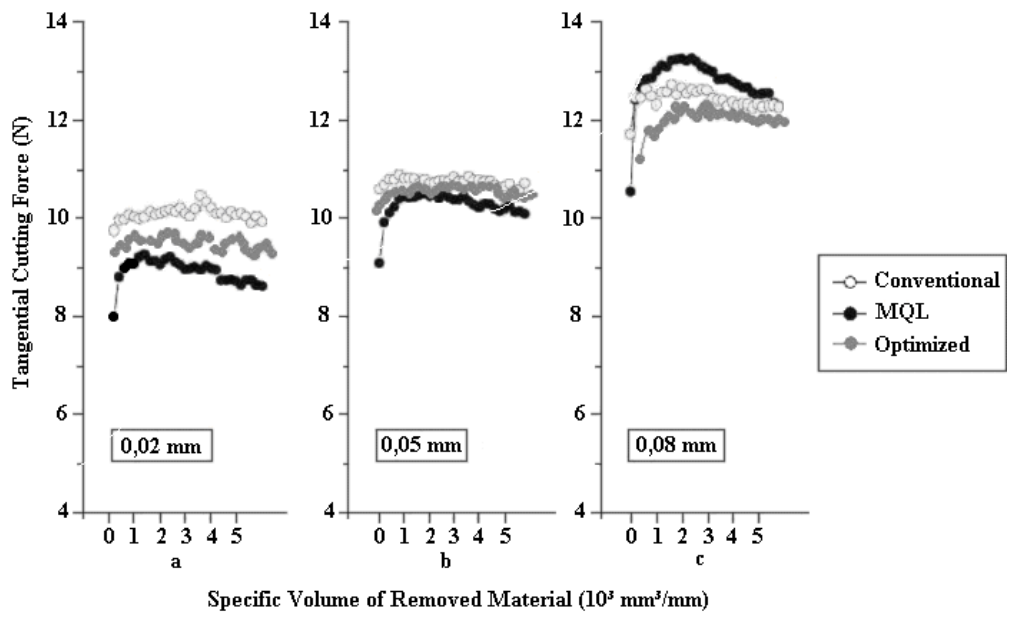

Figure 1: Tangential cutting force data - a) $0,02 \mathrm{~mm}$; b) $0,05 \mathrm{~mm}$; c) $0,08 \mathrm{~mm}$.

inefficient lubrication, the cutting zone cooling by the air flow is not able to minimize the excessive heat generation. In this case, the optimized method seems to be more efficient, because it allows better penetration in the cutting region and the removal of the excessive heat generated.

The optimized method showed good results due to its capability of cooling the cutting zone. This capability relies in the way the cutting fluid is applied. The high speed enables the fluid to penetrate the cutting zone, passing through the air barrier generated by the grinding wheel. The behaviour of this method throughout the increasing of the depth of cut is constant. The conventional method presents higher values, although its behaviour is constant too. These higher values are caused by the air barrier of the wheel, which the abundant flow of cutting fluid cannot surpass efficiently.

It is possible to conclude that increasing the depth of cut, the average values of tangential cutting force tend to increase as well. This is a consistent presumption, since an increase in the depth of cut produces a larger contact area between the grinding wheel and the workpiece, and although the number of grains in contact is also higher, the strength to remove more volume of material is increased, which consequently produces higher cutting forces.

Observing the cutting force behaviour, which varies along the experiment, it is possible to notice its inconstancy. It is due to the CBN super-abrasive grains friability. Along the process, the grains are worn out, losing their cutting edges, becoming flatter. This produces higher grinding forces, due to the decreasing of their cutting capacity and to the increasing of the drag process. Those worn out grains are released from the matrix, renewing the cutting edges. CBN grinding wheels have this capacity of self-sharpening, which strengthens their quality characteristic. 


\subsection{Surface roughness}

It is important to analyze the surface roughness, because the surface finishing affects the fatigue resistance of the workpiece significantly, when under strength. It is still linked to lubrication and depends mainly on the size of the abrasive grain present in the grinding wheel, on the dressing conditions and material removal rate [8].

Thus, due to the importance of surface roughness on ground workpiece, the average surface roughness $\left(R_{a}\right)$ was measured. In Figure 2, the values are given in micrometers, resulting from the arithmetic mean of all experiments with the same depth of cut.

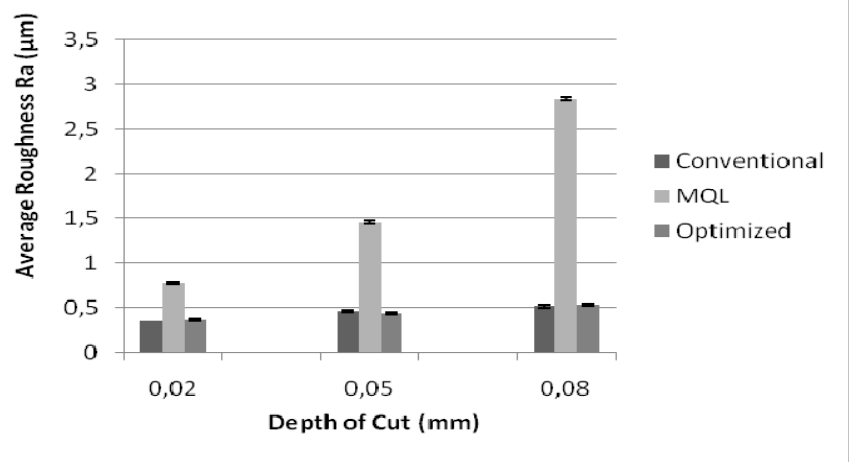

Figure 2: Comparison of roughness among the methods tested.

Figure 2 shows that all gathered in the conventional and optimized lubrication-cooling methods were below the ones obtained in the MQL method. At $0.08 \mathrm{~mm}$ depth of cut, surface roughness data in MQL are almost six times higher than the other values.

According to Malkin [2], the acceptable range for average roughness in a grinding process is from 0.2 to $1.6 \mu \mathrm{m}$. Therefore, all values obtained with the conventional and optimized methods are within the tolerance range, being quite satisfactory. The values are very similar, so it is possible to conclude that both methods remove chips away and guarantee the characteristic surface quality of the grinding process.

In the MQL, however, the data obtained at $0.08 \mathrm{~mm}$ depth of cut are out of the considered range, which disqualifies the process concerning its surface quality. At the best condition, $0.02 \mathrm{~mm}$, the values produced are higher than the ones produced by the other two methods. Nevertheless, MQL is a considerable alternative, even for finishing. For $0.05 \mathrm{~mm}$, MQL is in the limit of the considered range, which means the surface quality has not been kept. In this case and for $0.08 \mathrm{~mm}$ depth of cut, the MQL technique can be disqualified. 
The reasons of those variations can be explained. The conventional and optimized methods allow the removal of the chips in the cutting zone, improving the lubrication in the region and minimizing the friction between the tool and the workpiece. The MQL method, being inefficient in these tasks, makes the chips remain in the cutting zone, and the bigger the chip, more difficulty is to expel it, and worse surface quality is generated, which can be corroborated by the high values of surface roughness. The air flow is efficient in removing the chips produced with a $0.02 \mathrm{~mm}$ depth of cut. However, its efficiency decreases with the increase of the depth of cut, fact confirmed by the $0.05 \mathrm{~mm}$ value.

Although MQL requires lower cutting forces, the surface roughnesses are higher. The explanation to this fact consists of the grinding wheel topography. The CBN wheel lodges the thin chips produced in the MQL methods, resulting in lower forces and medium surface roughness. However, the increase in the depth of cut increases chip size, which makes more difficulty to settle the chips in the wheel porosity, which causes deplorable surface quality.

\subsection{Grinding wheel wear and $G$ ratio}

Grinding wheel wear is an extremely important variable in grinding, since the more the wear, the shorter the wheel life cycle. Figure 3 presents the diametric wear of the grinding wheel at each depth of cut, for the three types of lubricationcooling considered.

Figure 3 illustrates that the diametric wear of the grinding, wheel using the MQL technique, was lower at 0.02 and $0.05 \mathrm{~mm}$ depths of cut, whereas at $0.08 \mathrm{~mm}$, the value obtained was much higher than with the other two methods. These behaviours can also be observed with the cutting force data, since the higher the strength on the grinding wheel, the higher forces of cut needed and, consequently, higher tool wear.

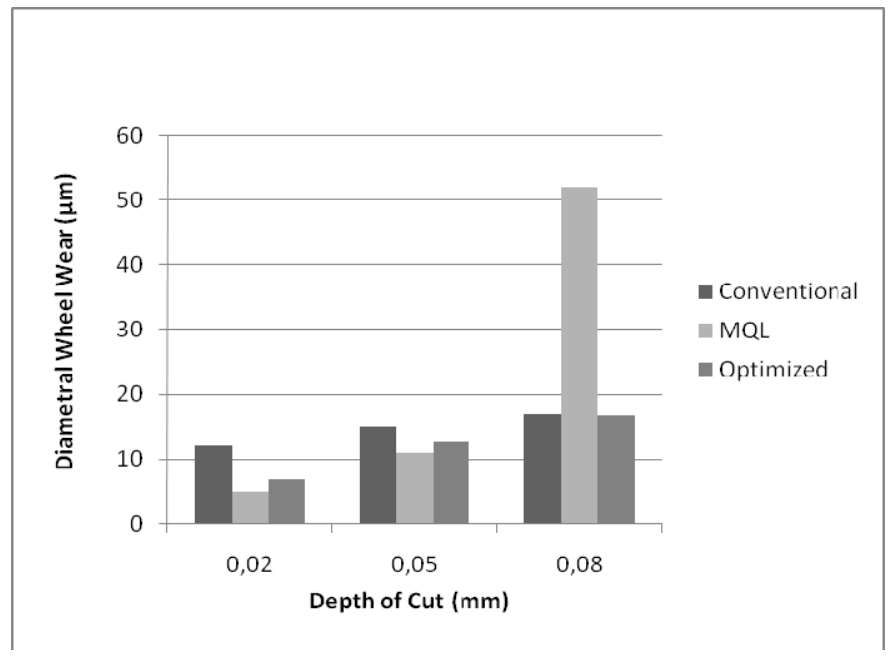

Figure 3: Grinding wheel wear comparison. 
Undoubtedly, the more the lubrication-cooling fluid penetrates in the cutting zone, less wear is produced by friction better is the removal of the generated heat. The porosity of the grinding wheel should be considered, since it is responsible for the accommodation of the chips produced, improving the access of the fluid to the cutting zone, with consequent improvement in lubrication, minimizing thus the wear by friction.

The grinding wheel used is a CBN grinding wheel with vitrified bond, which is less susceptible to erosive wear by chips, presenting a greater retention force on the abrasive grain and, consequently, minimizing the diametric loss of the grinding wheel.

In MQL at low depths of cut, parts of the chips mixed with atomized oil lodge in the wheel pores, allowing a portion of the lubricant to reach the cutting zone more effectively, decreasing the wear. This also allows the air flow to remove part of the heat produced, improving the cooling. So, even with this lodging condition producing a higher surface roughness, it contributes to lower tool wear.

However, when the generated chips becomes bigger, as when increasing the depth of cut, the pores are not able to accommodate them, and the air flow is not efficient to remove them from the cutting zone. Then, the chips damage the cutting and increase the abrasive strength of the tool on the workpiece, decreasing the lubrication capacity in the cutting zone, prejudicing the surface and wearing the wheel more intensely.

The optimized method also presents satisfactory results, being much more efficient than the conventional. The high speed of fluid application makes the removal of chips and excessive heat easier in the cutting zone, than the abundant fluid flow. It justifies why the optimized method showed better results, guaranteeing longer wheel life.

The $G$ Ratio is the ratio between the quantities of removed material and of worn material. This relation was obtained from the grinding wheel wear data.

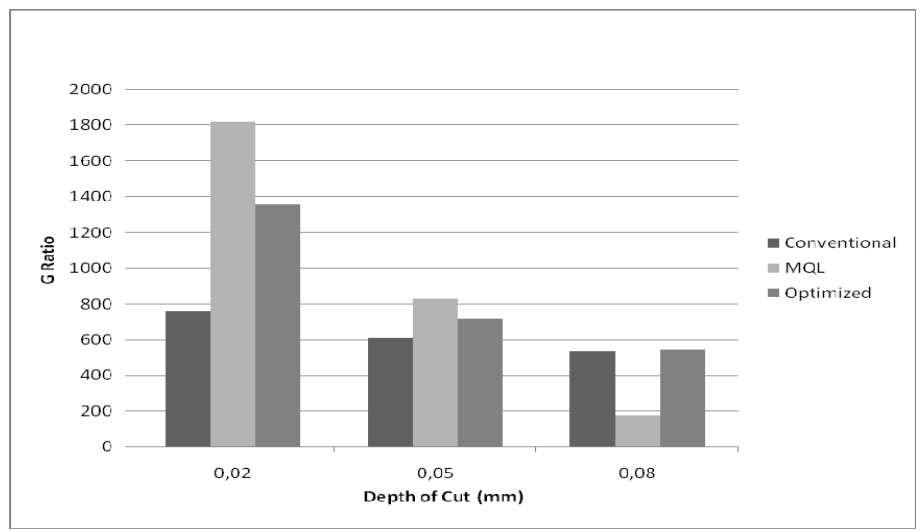

Figure 4: $G$ Ratio comparison among the lubrication-cooling methods analyzed. 
Figure 4 shows that the MQL is a good alternative concerning the application of lower depths of cut. The optimized method needs to be highlighted, since it shows that the fluid application in high speed is more efficient than the excessive fluid application in the conventional one. As the depth of cut increases, $G$ ratio values decrease, due to the increase of tool wear. For medium values, the MQL and the optimized method were the best. In this condition, however, the MQL presents high values for roughness, which is harmful for the process.

For higher depths of cut, the MQL showed to be ineffective in its functions. In this condition, the performances of the conventional and optimized methods e were similar, because the high quantity of heat is difficult to be removed, even with the fluid being applied at high speed.

\subsection{Microhardness}

Microhardness in the ground workpieces was also analyzed. This kind of analysis is made in deeper regions of the surface. After analyzing the gathered data, the graph in Figure 5 was produced. It also shows the reference value adopted, which is the microhardness before grinding.

The microhardness values obtained in the workpieces show that in all machining conditions and using the three methods of lubrication-cooling there is a loss concerning the reference. This is associated with the capacity of heat dissipation and cooling speed of the specimens. Such factors are determinant to define the granular structure obtained.

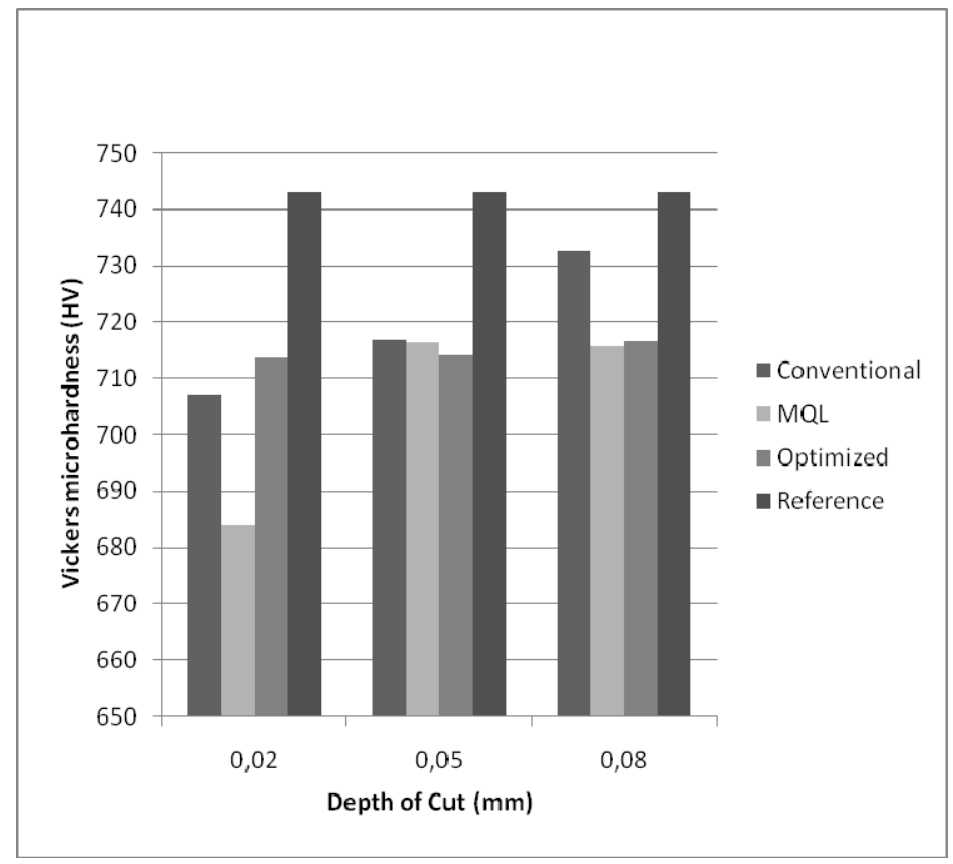

Figure 5: Vickers microhardness in the ground workpieces. 
Analyzing firstly the data gathered with $0.02 \mathrm{~mm}$ depth of cut, the loss obtained using the conventional method is smaller than the one using the MQL, but higher than the one with the optimized. This is consistent with the fact that MQL produces much more heat during cutting, while the excessive fluid and directed fluid jet of the conventional and optimized methods, respectively, remove more heat from the cutting zone. The ground workpieces under conventional lubrication-cooling lost around 5\% of their surface micro-hardness, compared to the reference. The ones ground by the optimized lost around $4 \%$, whereas by MQL, approximately $8 \%$.

At $0.05 \mathrm{~mm}$ depth of cut, the differences between the fluid application methods are very small, showing a very similar behaviour, which makes impossible to draw any conclusions about the microhardness tendency. On the other hand, there is a loss around $4 \%$, compared to a non-ground workpiece.

At $0.08 \mathrm{~mm}$ depth of cut, the values obtained by MQL are much lower than with conventional and optimized methods. This can be explained by the fact that MQL, in this condition, produces too much heat during cutting, consequently damaging the workpiece (with high surface roughness) and the tool (with enormous wear). In this grinding condition, microhardness loss by MQL was around $4 \%$ and approximately $1.5 \%$ by the conventional method. The optimized method was not very efficient to remove heat at high depths of cut, presenting behaviour similar to MQL and inferior to the conventional method.

Another interesting fact can be observed in figure 5. The microhardness loss in the most severe condition was smaller than the one obtained in the mildest condition. One of the reasons is that the latter took as many as three times longer than the most severe. Therefore, there was more exposure of the surface to constantly generated heat, although this was present in smaller values. Another explanation concerns to the performance of the CBN grinding wheel, developed for more severe work conditions, which allows better results under heavier machining conditions.

To sum up, it is possible to conclude that the heat generated during the grinding causes a reduction of the workpiece hardness, independently of the lubrication-cooling method utilized.

\section{Conclusions}

From data gathered with the experiments using three different methods of lubrication-cooling - conventional, optimized (with Webster nozzle) and MQL it is possible to conclude that, for plane grinding of AISI 4340 tempered and quenched steel, varying the depth of cut while keeping other grinding parameters constant, that:

- The tangential cutting force presented the smallest results in the MQL for 0.02 and $0.05 \mathrm{~mm}$ depth of cut, whereas for $0.08 \mathrm{~mm}$ in this method this variable suffers a high increase, being superior do the value obtained with the other methods. 
- The surface roughnesses of ground workpieces by conventional and optimized methods were always smaller than the ones presented in MQL. Surface roughness of workpieces ground when applying MQL is still in the acceptable range; however, for medium and high depths of cut, is remains on the limit or exceeds the values considered as acceptable. Under these conditions, the MQL produced low quality surfaces.

- The grinding wheel wear was smaller in MQL than in the other two methods. The optimized method is to be highlighted for presenting good performance at low and medium depths, whereas the conventional method is to be highlighted for higher values.

- The workpieces microhardnesses showed that in the three methods there was a loss of hardness due to the heat generated during the cutting process.

The MQL technique in grinding showed itself viable only when the removal of material is not severe. This way, it is possible to use MQL without considerable surface quality loss and with production gain, due to the longer life cycle of the grinding wheel. However, surface quality is impaired, producing rougher surfaces. For more removal of material, the MQL shows it is not an efficient lubrication-cooling method, damaging the workpiece finishing, wearing too much the tool.

The optimized method is to be highlighted for low and medium depths of cut values. It produces excellent surface finishing and small wheel wear. It shows that the application of cutting fluid with high speed is efficient in removing chips and the excessive heat from the grinding zone. For higher depths of cut, the conventional method is better indicated, although the resulting parameters were not satisfactory.

The selection of the method depends on several parameters. As long as equipment is available, the MQL is a very effective method for low material removal. The optimized method with Webster nozzle is a good alternative to the conventional method, because it makes the fluid application more effective, allowing a longer life cycle for the grinding tool.

This analysis was made varying the depth of cut and keeping other grinding parameters constant. A proposal for further researches is to vary other parameters, in order to attend industries demand, and analyze whether the cooling methods are efficient in maintaining process quality.

\section{References}

[1] Chen, X.; Rowe, W.B.; Mills, B.; et al., 1998, “Analysis and simulation of the grinding process. Part IV: effects of wheel wear", International Journal of Machine Tools \& Manufacture, Vol. 38, No. 1-2, pp. 41-49.

[2] Malkin, S., 1989, "Grinding Mechanisms e Grinding Temperatures and Thermal Damage”, In: Malkin, S. Grinding Technology: theory and 
applications of machining with abrasives, 1 ed., Chichester, Ellis Horwood Limited.

[3] Hassui, A.; Diniz, A. E., 2003, “Correlating surface roughness and vibration on plunge cylindrical grinding of steel”, International Journal of Machine Tools \& Manufacture, Vol. 43, pp. 855-862.

[4] Irani, R.A.; Bauer, R.J.; Warkentin, A., 2005, "A review of cutting fluid application in the grinding process", International Journal of Machine Tools \& Manufacture, Vol. 45, pp.1696-1705.

[5] Kopac, J.; Krajnik, P., 2006, "High-performance grinding - A Review", Journal of Materials Processing Technology, Vol. 175, pp. 278-284.

[6] Tawakoli, T.; Westkämper, E.; Rabiey, M. et al., 2007, "Influence of the type of coolant lubricant in grinding with CBN tools", International Journal of Machine Tools \& Manufacture, Vol. 47, pp.734-739.

[7] Ebbrell, S.; Woolley, N. H.; Tridimas, Y. D. et al., 2000, "The effects of cutting fluid application methods on the grinding process", International Journal of Machine Tools \& Manufacture, School of Engineering, Liverpool, U.K., Vol. 40, pp. 209-223.

[8] Webster, J. A., 1999, "Optimizing coolant application systems for high productivity grinding”, ABRASIVES Magazine, October/November, pp. 3441. 